\title{
Ulrich Blanché
}

\section{Black Box Banksy}

Im Zentrum des vorliegenden Aufsatzes steht die Kunstfigur und Black Box Banksy, mit seiner Geschichte und den an ihm vollzogenen Praktiken. Der britische Street Artist und ehemalige Graffiti Writer Banksy ist zugleich weltbekannt und (pseudo-)anonym. Sein Anonymsein in einer Welt, wo viele berühmt sein möchten, kann als vielleicht wichtigstes Banksy-Werk angesehen werden. Obwohl alte wie neue Medien vielfach über Banksy berichten, ist sein Gesicht und Aufenthaltsort sowie (zu weiten Teilen) seine Biografie nicht gesichert. Das ,System‘ hinter Banksy kommuniziert, zumindest mit Worten, nur digital. Wir wissen nicht, wie das komplexe digitale ,Artefakt“ Banksy funktioniert, aber aufgrund einer an uns angepassten Nutzerschnittstelle findet trotzdem ein erfolgreicher Austausch statt. Folgende Fragen stehen im Zentrum meines Beitrags: Wie und warum wurde die Black Box Banksy zwischen 1996 und 2005 langsam versiegelt? Was ist heute und damals seine Nutzeroberfläche? Wie wird das ,System‘ Banksy aufrechterhalten? Lässt sich die Black Box Banksy mit Hilfe des Dispositiv-Begriffs erkennen und öffnen?

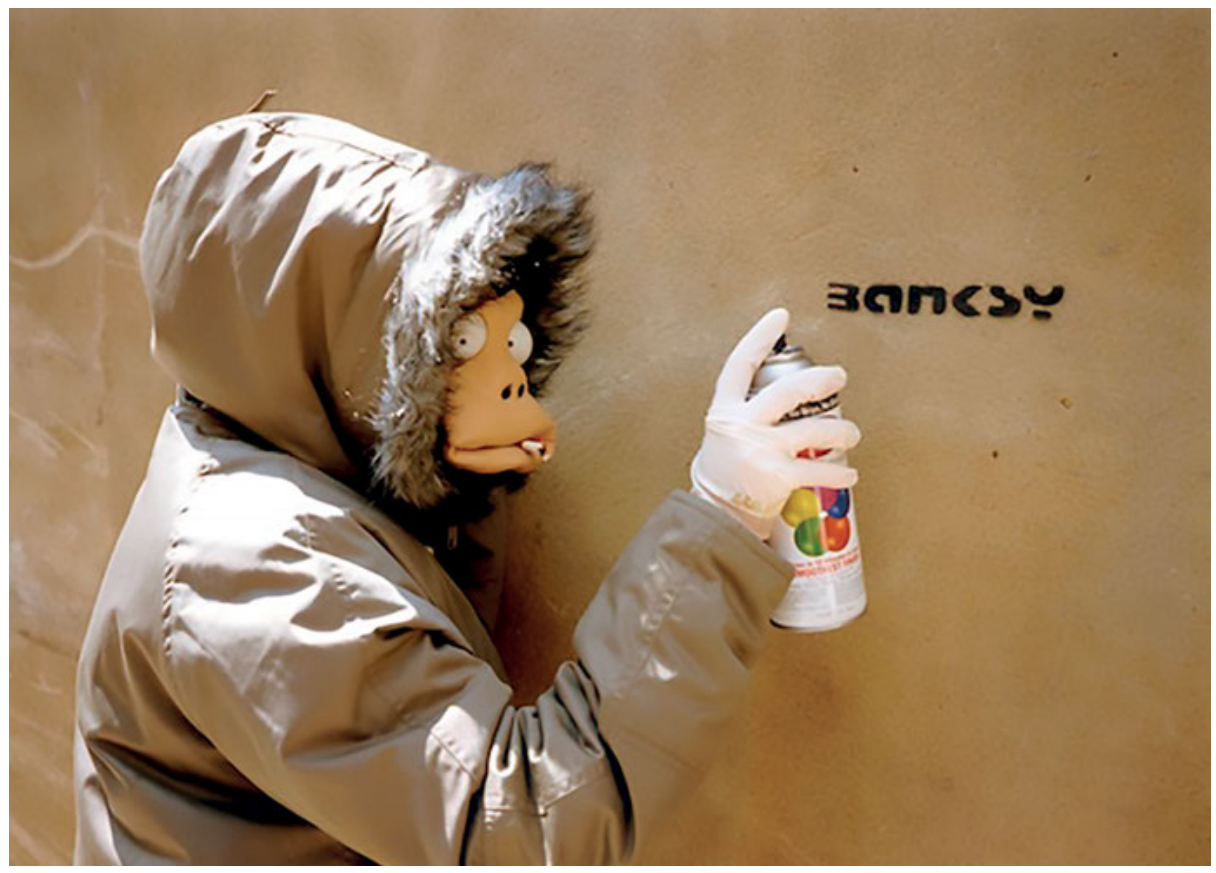

Abb. 1: Banksy, Monkey Mask Session, (Tag), London, 2003. 


\section{Banksy als Artefakt}

Der anonyme, britische Street Artist und ehemalige Graffiti Writer Banksy ist seit Mitte der 1990er Jahre aktiv. Banksy als Artefakt zu sehen erscheint zunächst vom Begriff her unpassend, da ein Artefakt im etymologischen und archäologischen Sinne ein von Menschen geformtes, gemachtes Ding ist, es sich bei Banksy jedoch um einen Künstler handelt, eine Person, eher einen Akteur. Da Banksy anonym ist, es sich nicht um seinen ,echten“ Namen handelt, er nicht als Person auftritt, sondern in erster Linie durch digitale Fotos und Texte (seinen Instagram Account, Email-Interviews, seine Website banksy.co.uk) mit der Öffentlichkeit kommuniziert und dies nicht als Individuum, sondern eher als Firma, als Marke, ist ,Banksy‘ zugleich auch etwas von Akteuren Erschaffenes und weiter Gelenktes, ein kulturelles digitales Artefakt: Darunter verstehe ich hier alles von Menschen Geschaffene, das Informationen über die Kultur der Erschaffer und ihrer Nutzer bereithält.

,Artefakte“ sind in vielen Bereichen, wo der Begriff verwendet wird, etwas Unerwünschtes, eine Störung, etwas Unbeabsichtigtes, Künstliches im negativen Sinne, etwa in der Diagnostik, Fotografie, Sozialforschung, Technik oder Computergrafik. Meist handelt es sich um eine visuelle Störung. Der Street Artist Banksy arbeitet bis heute mehrheitlich illegal, produziert also visuelle Störungen im Stadtraum. Den mit Banksy assoziierten Begriffen ,Graffiti‘, ,Vandalismus‘ und ,Schmiererei' lastet auch etwas Unbeabsichtigtes, Gedankenloses an, obwohl das Kunstwesen ,Banksy“ wie seine Werke zumindest künstlich, aus vielfacher Sicht auch künstlerisch hergestellt wurde. Das ,Arte‘ in ,Artefakt' meint eher ,künstlich“ ebenso wie sich Graffiti Writer und Street Artists oft nicht als Künstler verstehen, gerade nicht in einem Kunstkontext ausgebildet oder erfolgreich wurden. ${ }^{1}$ „I'm not so interested in convincing people in the art world that what I do is ,art““, sagt Banksy. „I'm more bothered about convincing people in the graffiti community that what I do is really vandalism. “2

\section{Banksy als Black Box}

Banksy wird im Folgenden als Black Box untersucht. Schon Ashby verstand unter Black Boxes nicht nur Dinge, sondern auch Menschen, ${ }^{3}$ ebenso Galloway. ${ }^{4}$ Der Ursprung des Begriffes ,Black Box‘ ist unklar. Wohl stammt er ursprünglich aus dem militärischen Jargon speziell der Fernmelde- und Sensortechnik. Man versteht darunter etwa ein defektes Bombenzielgerät ${ }^{5}$ oder ein Transportmittel, etwa eine kupfer-

\footnotetext{
1 Hoppe 2009.

2 Leopold 2010.

3 Ashby 1956, 86.

4 Galloway 2011, 273.

5 Ashby 1956, 86.
} 
ummantelte Black Box, in der ein neues Magnetron, das Radargeräte der Alliierten flexibler machen sollte, und die um 1940 von den Briten in einer Geheimmission in die USA gebracht wurde. ${ }^{6}$ Es bezeichnete auch erbeutetes Feindgerät der Deutschen etwa 1943/44, welches wegen der möglicherweise darin enthaltenen Sprengladung nicht geöffnet werden durfte. ${ }^{7}$ Und eine Black Box ist als ,schwarzer Kasten` auch Attribut des (Bühnen-)Zauberers. ${ }^{8}$ Die möglichen Ursprünge weisen auf jeden Fall auf etwas, von dem die Erschaffer nicht möchten, dass es geöffnet wird oder wo derjenige, der damit konfrontiert ist, aus Zeitmangel oder anderen Gründen beschließt, es nicht zu öffnen. ${ }^{9}$,Do not Open“ steht auch auf der Black Box von Flugschreibern. Dies gilt auch für den anonymen Vandalen Banksy: „I have no interest in ever coming out“. ${ }^{10}$ Wie die Black Box ist der Akteur hinter Banksy zwischen Zauberer und Guerilla angesiedelt. ,Black Box‘ ist vom Ursprung des Begriffs etwas mit Gewalt angeeignetes, wie eine Wand, auf der illegal Graffiti gesprüht wird, was im Graffiti-Jargon bombing genannt wird und ebenfalls militärischen Ursprungs ist, wie auch die Sprühdose im Französischen eine ,Bomb‘ ist. Black verweist auf unbekannt und dunkel, Box auf verschlossen, ein Ding, ein Container, etwas das einen Inhalt hat, seinen Inhalt und Funktion nicht gleich offenbart.

Banksy ist als Enigma zu verstehen, die prototypische Black Box - wir sollen über ihn nachdenken. Ihn zu „knacken“ wäre jedoch das Ende von Banksy wie wir ihn kennen (oder nicht kennen), da seine Werke auf Illegalität fußen, was ihm dann nicht mehr möglich wäre. Verschlüsselung ist Teil der Funktion der Black Box Banksy. Die Black Box ist ein Spekulationswerkzeug. Was Banksy von einem Artefakt im Sinne der Archäologie und von der Mehrzahl von Black Boxes, die man in die Hand nehmen kann, unterscheidet, was Banksy aber mit digitalen Artefakten gemeinsam hat, ist, dass er nicht ,fest-gestellt‘ werden kann. Ein archäologisches Artefakt kann nicht ,nachhause telefonieren'. Nur eine Black Box mit Funkverbindung kann etwa auf ihr Öffnen reagieren, zumindest auf neue Informationen, die die Erschaffer der Black Box zum Zeitpunkt des Erschaffens nicht hatten, oder zum letzten Zeitpunkt, wo die Erschaffer über die Black Box verfügten. Banksy ist wie ein Tier in freier Wildbahn. Man kann es beobachten, durchs Einfangen und Öffnen wird es etwas Anderes, es wird typischerweise zerstört. Durch das Betrachten der Black Box wird ein Handlungsdruck aufgebaut, wie durch das Betrachten von Banksys Werken an den dort kritisierten Sachverhalten durch die Betrachter etwas erkannt und geändert werden soll. Man kann die Werke Banksys auf den Nenner bringen, genauer hinzusehen, in Frage zu stellen.

6 Von Hilgers 2009, 127-145.

7 McCulloch 1974, 12.

8 Winkler 2014.

9 Ashby 1956, 86.

10 Fairey/Gastman 2006, 84. 
[I]n Kybernetik und Systemtheorie [bezeichnet Black Box] ein (möglicherweise sehr komplexes) System, von welchem im gegebenen Zusammenhang nur das äußere Verhalten betrachtet werden soll. Die innere Struktur mag bekannt sein; solche Kenntnis darf aber nicht benutzt werden (etwa weil ein Nachfolgemodell innen anders gebaut sein darf). Man beschränkt sich bei der Untersuchung und Beschreibung auf die Messung der Input-Output-Beziehungen. ${ }^{11}$

Banksy als Black Box zu untersuchen heißt also, nicht das Geheimnis lüften, sondern Input und Output untersuchen.

Die innere Struktur mag auch bei der Person Banksy bekannt sein. Wohl handelt es sich um den am 28. Juli 1973 in Bristol geborenen Robin Gunningham. ${ }^{12}$ Allein die Tatsache, dass das System Banksy diese Infos nicht freiwillig gibt, nicht verifiziert, sein Werk, der Output des Systems Banksy, jedoch dennoch gewinnbringend untersucht werden kann und ein großes Interesse daran besteht, es zu untersuchen, verweist darauf, dass es vielleicht im Falle eines jeden Künstlers unnötig ist, oder sein kann, oder sein sollte, seine Biografie zu kennen, zu wissen wie er angeblich funktioniert. Banksys Agieren als Black Box verweist darauf, dass Kunst mehr um Kunstwerke und deren Wirkung, weniger um Künstler gehen sollte. Die Mehrzahl der Medien widmen sich nicht der Kunst Banksys, sondern dem Rätsel um seine Person, die mehr Menschen interessiert. Als Einstieg mag dies gut sein, da das Spekulationswerkzeug Banksy im Idealfall dazu führt, dass die Person hinter Banksy zweitrangig wird. Stattdessen rückt das Spekulieren über Dinge, die Banksy schafft und behandelt, ins Zentrum. Persönliches wird sachlich.

Luhmann spricht von Black Boxes im Zusammenhang mit der doppelten Kontingenz:

Zum Unterbau, der im Theorem der doppelten Kontingenz vorausgesetzt ist, gehören hochkomplexe, sinnbenutzende Systeme, die für einander nicht durchsichtig und nicht kalkulierbar sind. Dies können psychische oder soziale Systeme sein. Wir müssen von deren Unterschied einstweilen absehen und sprechen deshalb von, black boxes'. Die Grundsituation der doppelten Kontingenz ist dann einfach: Zwei black boxes bekommen es, auf Grund welcher Umstände auch immer, miteinander zu tun. ${ }^{13}$

Banksy ist die eine Kontingenz. Diejenigen, die sich mit ihm und seinem Werk auseinandersetzen, sind die andere.

Die Motivation bei der Verwendung des Begriffs [Black Box] tendiert zu das Innere interessiert (jetzt) nicht, auch wenn er manchmal im Sinn von wir wissen es (sowieso) nicht verwendet wird. Diese Herangehensweise wird oft verwendet, um die Komplexität des Beobachtungsgegenstandes zu reduzieren. Das bewusste Weglassen von detaillierterer Information (Tiefeninformation) wird auch als Geheimnisprinzip bezeichnet. ${ }^{14}$

11 Winkler 2014.

12 Joseph 2008.

13 Luhmann 1993.

14 Wikipedia 2019. 
Da der Mensch hinter dem System ,Banksy“ nicht erkannt werden will - aus Datenschutzgründen oder um Strafverfolgung und Celebrity-Hype zu entgehen, jedoch auch, weil er es als unwichtig erachtet, als Mensch und nicht als Black Box in Erscheinung zu treten - muss jede Spekulation einseitig bleiben: ein vollständiges Verstehen einer anderen Kontingenz bleibt letztendlich unmöglich, die Multioptionalität eines Kunstwerkes oder Menschen auf Biografisches zu reduzieren, bleibt ein Abstempeln.

Wenn die Black Box Banksy geschlossen bleibt und nicht zu öffnen ist, muss stattdessen ihr In- und Output untersucht werden und die Frage gestellt werden, warum diese Black Box existiert: Kunst statt Künstler. Wer Michael Jackson oder Jeff Koons hinter ihrer artistic persona wirklich sind/waren, werden wir nie erfahren. Eventuell hat die Black Box Banksy gute Gründe, verschlossen zu sein, da sie eben darauf verweist, dass Manches, etwa die Person hinter Banksy, vielleicht gar nicht relevant ist - der Grund warum wir diese Black Box öffnen wollen jedoch schon. In einer überinformierten Gesellschaft, wo Aufmerksamkeit rares Gut ist, ist gerade das Vorenthalten von Informationen der einzige Weg, Aufmerksamkeit zu bekommen. Der Denkprozess, den Banksy beim Betrachter anstrebt anzustoßen, wird nicht durch Information, sondern durch deren Fehlen ausgelöst, was in einer Welt der Überinformation und Überkomplexität hilfreicher sein kann als viel über die Person hinter Banksy zu erfahren.

Banksy funktioniert eher wie ein Spiegel - weg vom Autor, hin zum Betrachter. Banksy spiegelt die Celebrity-Kultur, möchte das Gegenteil von Leuten sein, die berühmt dafür sind, berühmt zu sein: „I figure there are enough self-opinionated assholes trying to get their ugly little faces in front of you as it is. “15

\section{Black Box und Little Black Book: Wie und warum wurde die Black Box Banksy zwischen 1997 und 2005 langsam versiegelt?}

Bekannt wird Banksy ab 2000, zunächst in Bristol und London, um 2003 national in Großbritannien, ab 2006 schrittweise weltweit. Gleichzeitig wird er immer mehr zur Black Box. Von Beginn an suchte Banksy mit seinen Taten und Werken intensiv die Öffentlichkeit - dies bei gleichzeitigem Verbergen seiner Gesichtszüge und Verweigern der Preisgabe persönlicher Details über sein Privatleben. Er zeigte sein Werk und seinen Namen exzessiv, sich jedoch mit Maske.

Der Name ist ein Zwischending zwischen Werk und der Black Box. Anfangs machte Banksy dies, um Strafverfolgung zu entgehen (wie jeder Graffiti Writer) und sich gleichzeitig geheimnisvoller, damit interessanter zu machen, in den letzten Jahren

15 Fairey/Gastman 2006, 84. 
eher, weil es sein Markenzeichen ${ }^{16}$ und selbst ein politisches Statement geworden ist, keine gesicherte Biographie oder ein Gesicht zu haben. „The only conceivable way of unveiling a black box, is to play with it“". ${ }^{17}$ Statt einen Handlungsdruck durch das Geheimnisprinzip der Black Box beim Betrachter aufzubauen, soll positiver formuliert an dessen Spieltrieb appelliert werden. Das Risiko für das System ,Banksy‘ ist, dass es dabei kaputtgehen könnte, demaskiert wird. Zeigen und Verbergen fällt zusammen, es ist eine Aktivierung der Betrachter, die performanceartigen, illegalen Aktivitäten der Black Box Banksy zu knacken. Die Auseinandersetzung und der Nachvollzug der einstigen Herstellung der Black Box Banksy ist interessanter als deren Öffnung.

Im selben Zeitraum, in dem sich die Black Box Banksy langsam schließt, setzt sich das Internet weltweit immer mehr durch. Beides hängt zusammen. Zunächst war das Internet eher Spielplatz für Nerds und Bastler, langsam setzten sich große Firmen durch, es kam zur Monopolbildung. Das Internet hatte viel mit Selbermachen zu tun, Banksy richtete bereits 2000 eine Webseite ein. Er ist ein Online-Selfmademan, der unabhängig von klassischen Institutionen bekannt wurde. Seine Werke wurden weder aufgrund seiner Ausbildung (etwa an der renommierten Royal Academy) noch aufgrund von prestigeträchtigen Ausstellungsorten (wie der Tate, der Saatchi Gallery oder durch Sotheby's) bekannt, sondern auf der Straße und durch gezielt lancierte Infos an Presse und Medien - und nicht zuletzt durch seinen Webauftritt.

Dies manifestiert sich im Namen „Banksy“, einem Pseudonym, etwas in Selbstermächtigung selbst Gegebenes. Vielleicht verweist „Banksy“ auf den Klarnamen der Person hinter Banksy, Robin Gunningham, da die gun im Namen Bang macht. ${ }^{18}$ Auch ohne dieses Wissen ist ,Banksy‘ Teil des Langpseudonyms ,Robin Banks(y)`(,Banken ausrauben') eine alberne Anti-Kapitalismus-Kurzbotschaft à la ,Farin Urlaub‘, dem Sänger der deutschen Punk-Band ,Die Ärzte‘. Beide - Farin Urlaub und Banksy - kommen aus der analogen Punk-Bewegung, in der das Do it Yourself-Prinzip sehr wichtig war. Beim politischen Punk bedeutet das, ansteckend zu sein, andere dazu anzuregen, ebenfalls Gegebenes in Frage zu stellen, zugleich Individuum zu sein, Gegebenes wie etwa Namen nicht als gottgegeben anzunehmen, sondern spielerisch damit umzugehen. Neben Punk und der britischen Techno-Szene der 1990er Jahre zwischen illegalen free parties und Reclaim the Street, wo ebenfalls das Do it Yourself-Prinzip wichtig war, war der junge Graffiti Writer Banksy von Hiphop beeinflusst. Seine ersten drei kleinen Bücher haben ein mehrheitlich schwarzes Cover und sind handlich im Postkartenformat. Der hier als Black Box bezeichnete Banksy nennt sie Little Black Books. Ein ,Black Book` ist im analogen Graffiti-Kontext das persönliche Skizzenbuch eines Graffiti Writers und zugleich eine Art Poesiealbum oder Gästebuch, teils auch für Fotos, für befreundete oder bewunderte andere Writer, die einem auf Wunsch ihre Signatur oder eine Zeichnung darin hinterlassen. Sie sind nur für den zugänglich, der

16 Leopold 2010.

17 Thom 1984.

18 Blanché 2016, 205-212. 
Teil der Szene ist, fungieren zugleich als Stellvertreter der Black Box Banksy. Wird ein Black Book von der Polizei gefunden und einer Person zugeordnet, ist es für deren Strafverfolgung ähnlich folgenhaft wie wenn die Person in flagranti ertappt wurde.

Das teilhabende Do it Yourself-Prinzip galt bei Banksy zu Beginn nur für seine Werke, nicht für seine artistic persona, die in seiner Frühzeit mit seiner physischen Person noch zusammenfiel. Banksy wollte ursprünglich zum Selbermachen anregen, gab auf seiner Website und in Publikationen bis 2006 genaue Anweisungen zum Schablonen-selber-schneiden. Auf Graffiti- und Musik-Festivals war Banksy zu dieser Zeit noch persönlich anzutreffen, arbeitete gar als eine Art Auftrags- oder Schaumaler. Aufgrund der schrittweisen Veränderung des Internets, der damit geänderten Öffentlichkeit und öffentlichen Wahrnehmung entschied er sich offenbar, zur Black Box zu werden, da Fotos dieser Events jederzeit in die Hände etwa der Polizei fallen konnten.

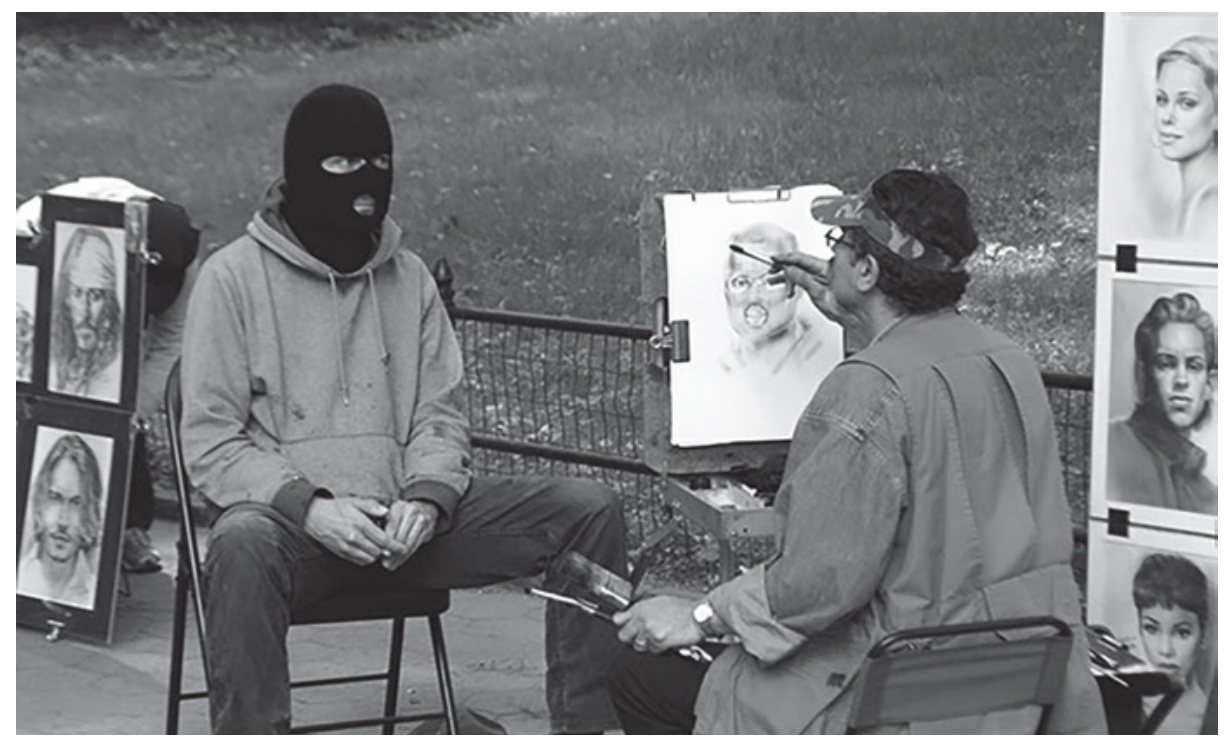

Abb. 2: Ein Mann mit einer Sturmmaske wird von einem Strassenmaler portraitiert. Abbildung auf der offiziellen FAQ-Seite Banksys.

\section{Was ist heute und damals Banksys Nutzeroberfläche und wie stabilisiert sich das System Banksy?}

Banksys Nutzeroberfläche ist zunächst seine Webseite, die er im Jahre 2000 einrichtet. Hier sind Fotos und Videos sowie begleitende Kommentare und Texte seiner Werke zu sehen, die oft wechseln, teils auch wiederkehren. Anfangs ist Banksy noch per Mail dort zumindest theoretisch erreichbar gewesen, später lange nicht mehr, in den letzten Jahren wieder, wobei klargemacht wird, dann man eher mit dem System 
,Banksy“ und seinen Mitarbeitern kommuniziert, nicht mit dem Meister selbst. Neben seiner Webseite lanciert Banksy schon vor dem Einrichten von banksy.co.uk Fotos und Berichte gezielt an die Presse, oft als Geheimnis, Puzzle oder Schnitzeljagd inszeniert. Im Jahr 2003 gibt er wohl sein letztes Face-to-Face Interview. ${ }^{19}$ Ab dem 1. Oktober 2013 löst sein Instagram-Profil seine Webseite als Hauptnutzeroberfläche langsam ab. Die Webseite bleibt bestehen, wird teils später auf Instagram geupdatet, auch mit anderem oder weniger Material. Auf Instagram verweigert Banksy Konventionen, antwortet nie auf Kommentare, abonniert niemanden, setzt keine Hashtags, ist zugleich mit 6,2 Millionen Abonnenten der auf Instagram führende Künstler weltweit. Teils vergingen Monate zwischen seinen Posts, seit 2017 postet er durchschnittlich einmal im Monat, mit großen Schwankungen. Das System Banksy wird durch seltene, in ihrer Unregelmäßigkeit doch regelmäßige Lebenszeichen am Laufen gehalten.

$\mathrm{Zu}$ Banksys Nutzeroberfläche gehören auch die Bilder von sich, die er an die Presse lanciert. Darauf ist eine wohl männliche erwachsene Person zu sehen, deren Gesicht immer abgewandt oder verdeckt ist: sei es, dass die Person eine (Affen-)Maske oder Papiertüte trägt, sich Hand, Buch, Sprühdose oder ein verpixeltes Portrait vors Gesicht hält oder schlicht den Hoodie ins Gesicht zieht. Ziel ist es, unterhaltend Neugier zu wecken, das Bedürfnis nach Bildern eines Autors zu befriedigen, dessen Werke weltbekannt sind und zugleich seine Anonymität und damit Operabilität der Black Box Banksy zu garantieren.

\section{Lässt sich die Black Box Banksy mit Hilfe des Dispositiv-Begriffs erkennen und öffnen?}

Foucault äußert sich zu seinem Dispositiv-Begriff 1977 in einem Interview. Er versteht darunter zugespitzt auf das knappe Format des Interviews

\footnotetext{
ein entschieden heterogenes Ensemble, das Diskurse, Institutionen, architekturale Einrichtungen, reglementierende Entscheidungen, Gesetze, administrative Maßnahmen, wissenschaftliche Aussagen, philosophische, moralische oder philanthropische Lehrsätze, kurz: Gesagtes ebenso wohl wie Ungesagtes umfasst. ${ }^{20}$
}

Die Elemente des Dispositivs „Banksy“ wären damit also die Diskurse um Banksy in Wort, Text und Bild, die „Institutionen“ wie Medien, Presse, Museen, Galerien, Polizei, Stadtverwaltung, Banksys Mitarbeiter. „Architekturale Einrichtungen“ sind etwa die Wände, auf denen Banksy sprüht und deren Bezüge zum Gesprühten, aber besonders auch die Werke selbst; „reglementierende Entscheidungen“ sind, etwa Gesetze

19 La Placa 2003.

20 Foucault 1978 [2000]. 
zu Maßnahmen zu Graffiti und Sachbeschädigung, zum Datenschutz, Vermummungsverbot, etc.; „wissenschaftliche Aussagen“ wie der vorliegende Text; „philosophische, moralische oder philanthropische Lehrsätze“, die Banksy verwendet, die auf Banksy anwendbar sind, die in seinen Werken zu finden sind, und vieles mehr.

Das Dispositiv selbst ist das Netz, das zwischen diesen Elementen geknüpft werden kann. Zweitens möchte [...][Foucault] mit dem Dispositiv gerade die Natur der Verbindung deutlich machen [...] Kurz gesagt gibt es zwischen diesen Elementen [...] ein Spiel von Positionswechseln und Funktionsveränderungen [...] Drittens verstehe ich unter Dispositiv eine Art von [...] Formation, deren Hauptfunktion zu einem gegebenen historischen Zeitpunkt darin bestanden hat, auf einen Notstand (urgence) zu antworten. Das Dispositiv hat also eine vorwiegend strategische Funktion. $^{21}$

Der Notstand bei Banksy war und ist erstens eine Reaktion auf den strukturellen Notstand im Kunstsystem, dass man als Künstler nur mittels des Marsches durch die Institutionen für die breite Masse wahrgenommen werden konnte - was jedenfalls für die Zeit gilt, bevor Künstler über das Internet Bekanntheit erlangen konnten (wie es z. B. Banksy als einem der ersten gelang). Zweitens ist Banksys Black-Box-Werden und -Sein eine Antwort auf den Notstand des mit der Bekanntheit einhergehenden Celebrity-Seins, das zu vermeiden war. Drittens hat Banksy durch sein Black-Box-Sein die Möglichkeit, eine Gegenöffentlichkeit für die vom ihm behandelten oder unterstützten, oft kontroversen Sujets zu schaffen, ,unverbrämt‘ dank Maske.

Laut Jürgen Link steht beim Dispositiv immer einem

,objektiven` instrumentellen Topik-Pol (maschineller Komplex, ,Klaviatur`) [...] ein ,subjektiver Verfügungs-Pol (am prägnantesten eine militärische Strategie) gegenüber. Mit Subjekt-Pol ist dabei also die Subjektivität des ,Disponierenden' gemeint, d. h. des Verfügenden über das Dispositiv, des Strategen, des Mächtigen. ${ }^{22}$

Als der Disponierende ist die Person hinter dem Dispositiv ,Banksy“ anzunehmen.

Lässt sich die Black Box Banksy also mit Hilfe des Dispositiv-Begriffs erkennen und öffnen? Die Frage ist zunächst, ob ein Öffnen das Ziel ist. Der Erkenntnisgewinn steht dem Öffnen gar gegenüber. Ein Öffnen würde in einer ,Fest-Stellung münden, ähnlich dem Schnitt durch ein lebendes Tier, man würde es zerstören. Ein Erkennen im Sinne eines vollständigen Erkennens, im Sinne eines Entlarvens, ist auch nicht das Ziel. Vielmehr ist das Ziel ein besseres Verstehen des In- und Outputs der Black Box Banksy, was jedoch einhergeht mit genauer Beobachtung der Black Box, der Art ihrer Verarbeitung des Inputs, etwa Zeugenbefragung, die einen früheren Zustand der Black Box glauben im offenen oder halboffenen Zustand gesehen zu haben, jedoch ohne sie zu zerstören. Der Input lässt sich bei Banksy mehrheitlich anhand des

21 Foucault 1978 [2000].

22 Link 2008. 
Outputs rekonstruieren, damit zugleich die Arbeitsweise der Black Box Banksy. Gerade wenn Banksy etwa seine Vorlagen und Inspirationsquellen entweder zitiert, bewusst falsch zitiert oder verschleiert, findet ein Erkenntnisgewinn beim Betrachter statt.

Die Antwort auf die Frage: ,Lässt sich die Black Box Banksy mit Hilfe des Dispositiv-Begriffs erkennen und öffnen?‘ wäre also: Erkennen ja - im Sinne von genauer sehen (lernen). Öffnen nein - wenn und weil Öffnen oft mit Zerstören zusammenfällt. Gerade im historischen Verlauf wird sichtbar, dass die Black Box Banksy mehrere Strategiewechsel durchmachte, das teils jeweils andere Akteure dahinter zu stecken scheinen und sich die Rolle des Disponierenden gewandelt hat, von einem Ein- bis Zweimann-Betrieb zu einer Firma. Die Strategiewechsel sind auf den Wechsel von analog zu digital zurückzuführen und dann auf den Wechsel von ,Banksy umwirbt Konsumenten seiner Botschaften` zu ,Konsumenten umwerben Banksy‘.

\section{Literaturverzeichnis}

Ashby, W. Ross (1956), An Introduction to Cybernetics, London.

Blanché, Ulrich (2016), Banksy. Urban Art in a Material World, Marburg.

Fairey, Shepard/Gastman, Roger (2006), „Banksy. The Man, The Myth, The Miscreant“, in: Swindle 8, 83-91.

Foucault, Michel (1978 [1977]), „Michel Foucault im Gespräch mit Jacques-Alain Miller und anderen Vertretern des Teams Psychoanalyse der Universität Paris-VIII“, in: ders., Dispositive der Macht. Über Sexualität, Wissen und Wahrheit (Neuaufl. 2000), Berlin, 119-125.

Galloway, Alexander R. (2011), „Black Box, Schwarzer Block“, in: Erich Hörl (Hg.), Die technologische Bedingung (suhrkamp taschenbuch wissen 1848), Berlin, 267-280.

Hilgers, Philipp von (2009), „Ursprünge der Black Box“, in: Philipp von Hilgers und Ana Ofak (Hgg.), Rekursionen. Von Faltungen des Wissens (Kulturtechniken 7), Berlin, 127-145.

Hoppe, Ilaria (2009), „Street Art und ,Die Kunst im öffentlichen Raum““, in: kunsttexte.de, Gegenwart 2009 (1), edoc.hu-berlin.de/kunsttexte/2009-1/hoppe-ilaria-6/PDF/hoppe.pdf (Stand: 3.7.2019).

Joseph, Claudia (2008), „Graffiti artist Banksy unmasked ... as a former public schoolboy from middle-class suburbia“, in: Daily Mail 12.7.2008, www.dailymail.co.uk/femail/article-1034538/ Graffiti-artist-Banksy-unmasked---public-schoolboy-middle-class-suburbia.html (Stand: 3.7.2019).

La Placa, Joe (2003), „London Calling“, in: Artnet 25.8.2003, http://www.artnet.com/magazine/ reviews/laplaca/laplaca8-25-03.asp (Stand: 3.7.2019).

Leopold, Shelley (2010), „Banksy Revealed?“, in: LA Weekly, 8.4.2010, www.laweekly.com/ banksy-revealed/ (Stand: 3.7.2019).

Link, Jürgen (2008), „Dispositiv“, in: Clemens Kammler, Rolf Parr u. Ulrich Johannes Schneider (Hgg.), Foucault-Handbuch. Leben - Werk - Wirkung, Stuttgart et al., 238.

Luhmann, Niklas (1993 [1984]), Soziale Systeme. Grundriß einer allgemeinen Theorie (suhrkamp taschenbuch wissen 666), Frankfurt a. M.

McCulloch, Warren (1974), „Recollections of the Many Sources of Cybernetics“ (Manuskript 1969), in: ASC Forum 6 (2), 5-16. 
Thom, René (1984), Mathematical Models of Morphogenesis, Chichester.

Wikipedia (2019), „Black Box (Systemtheorie)“, Version vom 27. Juni 2019, https://de.wikipedia.org/ wiki/Black_Box_(Systemtheorie) (Stand: 3.7.2019).

Winkler, Hartmut (2014), Black Box und Black Boxing - Zur Einführung. (Unveröffentlichtes Manuskript des Vortrags im Graduiertenkolleg ,Automatismen‘, Universität Paderborn, 14.10.2014), homepages.uni-paderborn.de/winkler/gk-black.pdf (Stand: 3.7.2019).

\section{Bildnachweise}

Abb. 1: Foto von James Pfaff.

Abb. 2: Offizelle FAQ-Seite Banksys, https://banksy.co.uk/faq.asp (Stand: 9.12.2019). 
